\title{
A Minimally Fine-Tuned Supersymmetric Standard Model
}

\section{David Tucker-Smith*}

Williams College, USA

E-mail: dtuckers@williams.edu

\begin{abstract}
I describe supersymmetric theories in which the correct scale for electroweak symmetry breaking is obtained without significant fine-tuning. In these theories, supersymmetry breaking occurs at a low scale of order $100 \mathrm{TeV}$, and is transmitted to the supersymmetric standard-model sector through standard-model gauge interactions. The Higgs sector contains two Higgs doublets and a singlet field, with a superpotential that takes the most general form allowed by gauge invariance. I discuss an explicit model constructed in 5D warped space with supersymmetry broken on the infrared brane, and present the results of a detailed analysis of electroweak symmetry breaking for this model. A new candidate for dark matter is also proposed, which arises from the extended Higgs sector of the model.
\end{abstract}

International Europhysics Conference on High Energy Physics

July 21st - 27th 2005

Lisboa, Portugal

* Speaker. 


\section{Introduction}

Low-energy supersymmetry stabilizes the electroweak scale by enforcing a high degree of cancellation among the radiative contributions to the Higgs potential, and also leads to a successful prediction for the low-energy gauge couplings through gauge coupling unification [1]. However, the fact that neither a light Higgs nor superparticles were discovered at LEP II requires relatively large superpartner masses, which in turn produce a large negative contribution to the Higgs masssquared at loop level. This typically means that a significant fine-tuning among the parameters of the theory is required to achieve realistic electroweak symmetry breaking.

Here I summarize Ref. [2], in which my collaborators and I presented a supersymmetric theory in which this fine-tuning problem is essentially absent, while the most attractive features of low-energy supersymmetry are preserved. An important ingredient is an extra contribution to the physical Higgs-boson mass, beyond those given in the minimal supersymmetric standard model (MSSM). This contribution arises from a coupling between the MSSM Higgs doublets and a singlet superfield $S$, as in the NMSSM [3]. The form of the superpotential we consider, however, differs from that of the NMSSM, and so there are new parameter regions to explore. Once we adopt a low mediation scale for supersymmetry breaking and eliminate unified mass relations among the superparticle masses, we find regions that have only a very mild fine-tuning.

\section{The Supersymmetric Fine-Tuning Problem}

I'll first review the sources of fine-tuning in conventional supersymmetric theories, to motivate the model-building that follows. The leading negative contribution to the Higgs-boson masssquared parameter in the MSSM comes from top quark and squark loops, and is approximately

$$
m_{h}^{2} \simeq-\frac{3 y_{t}^{2}}{4 \pi^{2}} m_{\tilde{t}}^{2} \ln \left(M_{\text {mess }} / m_{\tilde{t}}\right),
$$

where $y_{t}$ is the top Yukawa coupling, $m_{\tilde{t}}$ represents the top square masses, and $M_{\text {mess }}$ is the scale at which supersymmetry breaking is mediated to the MSSM sector. If $M_{m e s s}$ is near the Planck scale, as in the minimal supergravity scenario [4], then this contribution is large, $m_{h}^{2} \simeq(500 \mathrm{GeV})^{2}$ even for $m_{\tilde{t}}^{2} \simeq(300 \mathrm{GeV})^{2}$. For moderately large values of $\tan \beta$, the electroweak scale is determined at tree level by

$$
\frac{M_{Z}^{2}}{2} \simeq-m_{h}^{2}-|\mu|^{2}
$$

and the cancellation required between the two terms on the right-hand side is therefore at the level of a few percent. The same conclusion is reached by a more careful renormalization group analysis [5].

If we make the $\log$ of Eqn. (2.1) small by lowering $M_{\text {mess }}$, the most natural way to preserve the supersymmetric desert while obtaining flavor-universal superparticle masses is by mediating supersymmetry breaking by the standard model gauge interactions. Assuming that supersymmetry is broken in a sector that respects a global $S U(5)$ symmetry, the ratio of the top-squark mass to the right-handed selectron mass is then

$$
\frac{m_{\tilde{t}}^{2}}{m_{\tilde{e}}^{2}} \simeq \frac{(4 / 3) g_{3}^{4}}{(3 / 5) g_{1}^{4}} \simeq(7 \sim 9)^{2} .
$$


The LEP II bound $m_{\tilde{e}} \gtrsim 100 \mathrm{GeV}$ then implies $m_{\tilde{t}} \gtrsim 700 \mathrm{GeV}$. Meanwhile, the bound on the righthanded selectron mass combined with the requirement that the standard-model gauge couplings do not hit a Landau pole below the unification scale tells us that the smallest $M_{\text {mess }}$ can be in this framework is roughly $20 \mathrm{TeV}$. These results again imply fine-tuning at the level of a few percent. In fact, even if the unified mass relation of Eqn. (2.3) is not satisfied, the LEP II lower bound on the mass of the lightest CP-even Higgs boson - which is $114 \mathrm{GeV}$ in most of the parameter space [6] by itself requires $m_{\tilde{t}} \gtrsim(800-1000) \mathrm{GeV}$ for natural values of the top-squark mass parameters [7], and so the fine-tuning persists.

\section{Basic Structure of the Theory}

In light of the fine-tuning discussion above, we require that our model feature the following properties: (i) the messenger scale should be low, $M_{\text {mess }} \sim 10-100 \mathrm{TeV}$, (ii) there should be an additional contribution to the mass of the light Higgs, beyond that given in the MSSM, and (iii) the superparticle spectrum must not obey unified mass relations, as would arise e.g. if the supersymmetry-breaking sector respected an $S U(5)$ symmetry.

We want to implement these features in such a way that the successful prediction associated with gauge coupling unification is preserved, and without reintroducing the supersymmetric flavor problem. Finally, if possible, we want a viable dark-matter candidate to emerge - given the smallness of $M_{\text {mess }}$, the LSP will be the gravitino, with mass in the $\mathrm{eV}$ range, so ordinary neutralino dark matter is not an option.

These considerations lead us to assume that supersymmetry is broken dynamically at a low scale $\Lambda \sim M_{\text {mess }} \sim 10-100 \mathrm{TeV}$. We take the dynamical supersymmetry breaking (DSB) sector to be charged under the standard model gauge group, so that supersymmetry breaking is communicated to the MSSM sector through gauge interactions, ensuring flavor universality of the squark and slepton masses. To preserve coupling unification, we assume that the DSB sector respects an approximate global $S U(5)$ symmetry that is explicitly broken only by the standard model gauge couplings. However, we take the global symmetry to be broken spontaneously by the strong dynamics of the DSB sector, at the scale $\Lambda$. This spontaneous breaking means that there is no reason for the superpartner masses to obey $S U(5)$ mass relations, as desired. Above $\Lambda$, the contributions to the gauge coupling running from the DSB sector is still approximately $S U(5)$-symmetric, and coupling unification remains intact.

If the DSB sector satisfies certain properties, the AdS/CFT correspondence [8, 9] suggests that we can adopt a dual description in 5D AdS spacetime truncated by what we call the Planck and $\mathrm{TeV}$ branes. The standard model gauge fields propagate in the 5D bulk, while quarks, leptons, and the Higgs sector are localized to the Planck brane. Breaking supersymmetry by the IR dynamics of the DSB sector corresponds in 5D to localizing supersymmetry breaking on the TeV brane. To preserve the successful MSSM prediction for the low-energy gauge couplings, the 5D bulk must respect $S U(5)$, which is broken to the standard model subgroup at the Planck brane by boundary conditions. In the 4D picture this corresponds to the fact that the DSB sector possesses a global $S U(5)$ symmetry, of which only the standard model subgroup is gauged. The spontaneous breaking of the global $S U(5)$ in the 4D picture corresponds to having boundary conditions that break $S U(5)$ to the standard model subgroup on the $\mathrm{TeV}$ brane. 
To get an extra contribution to the light Higgs mass, we introduce a singlet superfield $S$ on the Planck brane, and the superpotential coupling $S_{4} H_{d}$. It is important for $S$ feel supersymmetry breaking somewhat strongly, and so we imagine that supersymmetry breaking is mediated to the Higgs sector through a different set of bulk singlet $P$ superfields that couple directly to the DSB sector. We find that it is possible to construct the model so that it respects a $Z_{2}$ symmetry under which only the $P$ fields are odd, and so the lightest $P$-type particle is a stable dark-matter candidate.

Taking just a single $P$ field for illustration, the superpotential contains the terms

$$
W_{H}=\lambda S H_{u} H_{d}+\frac{\eta}{2} S P^{2},
$$

The interactions of $P$ with the DSB sector induce an effective supersymmetric mass term for $P$, along with supersymmetry-breaking terms including a soft mass-squared, a $B$-term, and an $S P^{2}$ $A$-term. Given our assumption about $M_{\text {mess }}$, we expect the typical energy scale of the coefficients of these terms to be roughly $10 \mathrm{TeV}$ or so. Loops of $P$ fields generate effective superpotential terms and soft supersymmetry-breaking terms for $S$. In particular, after integrating out the $P$ multiplet we get

$$
W_{\mathrm{eff}, S}=L_{S}^{2} S
$$

and

$$
\mathscr{L}_{\text {soft }, S}=-\left(L_{S}^{2} C_{S} S+\lambda A_{\lambda} S H_{u} H_{d}+\text { h.c. }\right)-m_{S}^{2}|S|^{2},
$$

where $L_{S}^{2}, L_{S}^{2} C_{S}, A_{\lambda}$ and $m_{S}^{2}$ are coefficients. Note that there is a linear term in $S$ in the potential, a fact which becomes important when we study electroweak symmetry breaking. We also consider the possibility that there are additional terms in the Higgs-sector superpotential, namely

$$
\delta W=\frac{M_{S}}{2} S^{2}+\frac{\kappa}{3} S^{3}
$$

where $M_{S}$ is a parameter of order the weak scale. The second term could be generated through a superpotential coupling of the form $S^{2} X$, if $X$ is a bulk field that receives a weak-scale VEV through its interactions with the DSB sector.

\section{Electroweak Symmetry Breaking and Results for Fine-Tuning}

Once we include soft masses-squared for the Higgs doublets, which come from gauge and top loops, we can study electroweak-symmetry breaking and fine-tuning in this setup. Here I simply summarize the results; the details of our analysis and a more extensive discussion of the results and the phenomenology of this setup can be found in [2].

A renormalization group analysis indicates that we can take $\lambda$ to be as large as 0.8 at low energies, and allow $\tan \beta$ as small as 1.7 (for $\lambda=0.8$ ). Also, by considering how they are generated radiatively, we obtain approximate upper bounds on the parameters $L_{S}^{2}, L_{S}^{2} C_{S}, A_{\lambda}$ and $m_{S}^{2}$. It turns out to be advantageous to take $m_{S}^{2}>0$ to stabilize the VEV of $S$; the linear term in the potential for $S$ ensures that it is unstable at $\langle S\rangle=0$. Defining the fine-tuning parameter as the fractional change of the weak scale in response to fractional changes of the fundamental parameters $a_{i}$ of the theory, $\Delta=\min _{i}\left|\left(a_{i} / M_{Z}^{2}\right)\left(\partial M_{Z}^{2} / \partial a_{i}\right)\right|[10]$, we find after minimizing the potential that the fine-tuning can be as mild as $\sim 20 \%$ for this setup. Three sample parameter points, along with the resulting 
Higgs and superparticle spectrum and the associated fine-tuning, are shown in the tables below. The parameter $k^{\prime}$ is the scale of the TeV brane, which is closely related to the scale $\Lambda$ of the DSB sector. The $\tilde{\Delta}^{-1}$ quantity is a different (and better) measure of fine-tuning that takes into account the generic sensitivity of the weak scale to the various parameters; see [2] for details. We find that the parameter regions with reduced fine-tuning require the superparticles to be relatively light, so this scenario would lead to a plethora of discoveries at the LHC.

\begin{tabular}{|c|c|c|c|}
\hline & $\mathrm{A}$ & $\mathrm{B}$ & $\mathrm{C}$ \\
\hline$\lambda$ & 0.8 & 0.8 & 0.8 \\
$M_{S}$ & 317 & 0 & 0 \\
$\kappa$ & 0 & 0.2 & 0 \\
\hline$\left[L_{S}^{2}\right]^{1 / 2}$ & -85 & 203 & 243 \\
{$\left[L_{S}^{2} C_{S}\right]^{1 / 3}$} & -523 & -464 & -535 \\
{$\left[A_{\lambda}\right]$} & -21 & 21 & 25 \\
{$\left[m_{S}^{2}\right]^{1 / 2}$} & 808 & 683 & 787 \\
{$\left[m_{H_{u}}^{2}\right]^{1 / 2}$} & -106 & -102 & -124 \\
{$\left[m_{H_{d}}^{2}\right]^{1 / 2}$} & 192 & 192 & 193 \\
$k^{\prime}$ & $8 \times 10^{4}$ & $7 \times 10^{4}$ & $7 \times 10^{4}$ \\
\hline$\Delta^{-1}$ & $19 \%$ & $18 \%$ & $12 \%$ \\
$\tilde{\Delta}^{-1}$ & $26 \%$ & $24 \%$ & $22 \%$ \\
\hline
\end{tabular}

\begin{tabular}{|c|c|c|c|}
\hline & $\mathrm{A}$ & $\mathrm{B}$ & $\mathrm{C}$ \\
\hline$\tilde{g}^{ \pm}$ & 305 & 307 & 328 \\
\hline$\chi_{1}^{ \pm}$ & 115 & 121 & 150 \\
$\chi_{2}^{ \pm}$ & 297 & 314 & 332 \\
\hline$\chi_{1}^{0}$ & 103 & 88 & 56 \\
$\chi_{2}^{0}$ & 193 & 162 & 132 \\
$\chi_{3}^{0}$ & 288 & 221 & 200 \\
$\chi_{4}^{0}$ & 353 & 262 & 263 \\
$\chi_{5}^{0}$ & 365 & 321 & 336 \\
\hline$H_{1}^{0}$ & 140 & 134 & 130 \\
$H_{2}^{0}$ & 298 & 304 & 332 \\
$H_{3}^{0}$ & 872 & 718 & 802 \\
\hline$P_{1}^{0}$ & 305 & 315 & 343 \\
$P_{2}^{0}$ & 888 & 687 & 799 \\
\hline$H^{ \pm}$ & 288 & 293 & 323 \\
\hline$\tilde{u}_{L}$ & 309 & 317 & 337 \\
$\tilde{u}_{R}$ & 294 & 281 & 289 \\
$\tilde{d}_{L}$ & 315 & 322 & 341 \\
$\tilde{d}_{R}$ & 272 & 270 & 285 \\
$\tilde{e}_{L}$ & 195 & 195 & 196 \\
$\tilde{e}_{R}$ & 203 & 153 & 105 \\
$\tilde{\nu}_{L}$ & 186 & 187 & 188 \\
\hline$\tilde{t}_{1}$ & 221 & 214 & 212 \\
$\tilde{t}_{2}$ & 385 & 387 & 404 \\
\hline
\end{tabular}

Figure 1: For three sample points, values for the parameters of the model (left) and masses of the Higgs bosons and superparticles (right). In the table to the left, $[X]^{n} \equiv \operatorname{sgn}(X) \cdot|X|^{n}$, and all masses are in GeV.

I would like to thank Z. Chacko and Y. Nomura for the collaboration on which this talk was based, and the Research Corporation for support.

\section{References}

[1] S. Dimopoulos and H. Georgi, Nucl. Phys. B 193, 150 (1981); N. Sakai, Z. Phys. C 11, 153 (1981); S. Dimopoulos, S. Raby and F. Wilczek, Phys. Rev. D 24, 1681 (1981).

[2] Z. Chacko, Y. Nomura and D. Tucker-Smith, Nucl. Phys. B 725, 207 (2005) [arXiv:hep-ph/0504095].

[3] H. P. Nilles, M. Srednicki and D. Wyler, Phys. Lett. B 120, 346 (1983); J. M. Frere, D. R. T. Jones and S. Raby, Nucl. Phys. B 222, 11 (1983); J. P. Derendinger and C. A. Savoy, Nucl. Phys. B 237, 307 (1984); J. R. Ellis, J. F. Gunion, H. E. Haber, L. Roszkowski and F. Zwirner, Phys. Rev. D 39, 844 (1989).

[4] A. H. Chamseddine, R. Arnowitt and P. Nath, Phys. Rev. Lett. 49, 970 (1982); R. Barbieri, S. Ferrara and C. A. Savoy, Phys. Lett. B 119, 343 (1982); L. J. Hall, J. Lykken and S. Weinberg, Phys. Rev. D 27, 2359 (1983).

[5] P. H. Chankowski, J. R. Ellis, M. Olechowski and S. Pokorski, Nucl. Phys. B 544, 39 (1999) [arXiv:hep-ph/9808275]; M. Bastero-Gil, G. L. Kane and S. F. King, Phys. Lett. B 474, 103 (2000) [arXiv:hep-ph/9910506]; J. A. Casas, J. R. Espinosa and I. Hidalgo, JHEP 0401, 008 (2004) [arXiv:hep-ph/0310137]. 
[6] LEP Higgs Working Group Collaboration, arXiv:hep-ex/0107030.

[7] See, for example, M. Carena, M. Quiros and C. E. M. Wagner, Nucl. Phys. B 461, 407 (1996) [arXiv:hep-ph/9508343]; H. E. Haber, R. Hempfling and A. H. Hoang, Z. Phys. C 75, 539 (1997) [arXiv:hep-ph/9609331].

[8] J. M. Maldacena, Adv. Theor. Math. Phys. 2, 231 (1998) [Int. J. Theor. Phys. 38, 1113 (1999)] [arXiv:hep-th/9711200]; S. S. Gubser, I. R. Klebanov and A. M. Polyakov, Phys. Lett. B 428, 105 (1998) [arXiv:hep-th/9802109]; E. Witten, Adv. Theor. Math. Phys. 2, 253 (1998) [arXiv:hep-th/9802150].

[9] N. Arkani-Hamed, M. Porrati and L. Randall, JHEP 0108, 017 (2001) [arXiv:hep-th/0012148]; R. Rattazzi and A. Zaffaroni, JHEP 0104, 021 (2001) [arXiv:hep-th/0012248].

[10] R. Barbieri and G. F. Giudice, Nucl. Phys. B 306, 63 (1988). 